\title{
Saturated ablation in metal hydrides and acceleration of protons and deuterons to keV energies with a soft-x-ray laser
}

J. Andreasson, ${ }^{1}$ B. Iwan, ${ }^{1}$ A. Andrejczuk, ${ }^{2}$ E. Abreu,,${ }^{1, *}$ M. Bergh, ${ }^{3}$ C. Caleman,,${ }^{4} \dagger$ A. J. Nelson, ${ }^{5}$ S. Bajt ${ }^{6}$ J. Chalupsky, ${ }^{7}$ H. N. Chapman, ${ }^{8,9}$ R. R. Fäustlin, ${ }^{6}$ V. Hajkova, ${ }^{7}$ P. A. Heimann, ${ }^{10}$ B. Hjörvarsson, ${ }^{11}$ L. Juha, ${ }^{7}$ D. Klinger, ${ }^{12}$ J. Krzywinski, ${ }^{14}$ B. Nagler, ${ }^{14}$ G. K. Pálsson, ${ }^{11}$ W. Singer, ${ }^{6}$ M. M. Seibert, ${ }^{1}$ R. Sobierajski, ${ }^{12,13}$ S. Toleikis, ${ }^{6}$ T. Tschentscher, ${ }^{6, \ddagger}$ S. M. Vinko, ${ }^{15}$ R. W. Lee, ${ }^{5,14}$ J. Hajdu, ${ }^{1}$ and N. Tîmneanu ${ }^{1, \S}$

${ }^{1}$ Department of Cell and Molecular Biology, Uppsala University, Box 596, SE-75124 Uppsala, Sweden

${ }^{2}$ Faculty of Physics, University of Bialystok, Lipowa Str. 41, PL-15424 Bialystok, Poland

${ }^{3}$ Swedish Defence Research Agency, SE-16490 Stockholm, Sweden

${ }^{4}$ Physik Department E17, Technische Universität München, James-Franck-Strasse, DE-85748 Garching, Germany

${ }^{5}$ Lawrence Livermore National Laboratory, 7000 East Avenue, Livermore, California 94550, USA

${ }^{6}$ Deutsches Elektronen-Synchrotron DESY, Notkestrasse 85, DE-22603 Hamburg, Germany

${ }^{7}$ Institute of Physics ASCR, Na Slovance 2, CZ-18221 Prague 8, Czech Republic

${ }^{8}$ Center for Free-Electron Laser Science, DESY, Notkestraße 85, DE-22607 Hamburg, Germany

${ }^{9}$ Department of Physics, University of Hamburg, Luruper Chaussee 149, DE-22761 Hamburg, Germany

${ }^{10}$ Lawrence Berkeley National Laboratory, 1 Cyclotron Road, California 94720, USA

${ }^{11}$ Department of Physics, Uppsala University, Box 530, SE-75121 Uppsala, Sweden

${ }^{12}$ Institute of Physics, Polish Academy of Sciences, Al. Lotnikow 32/46, PL-02668 Warsaw, Poland

${ }^{13}$ FOM-Institute for Plasma Physics Rijnhuizen, Edisonbaan 14, NL-3430 Nieuwegein, The Netherlands

${ }^{14}$ SLAC National Accelerator Laboratory, 2575 Sand Hill Road, Menlo Park, California 94025, USA

${ }^{15}$ Department of Physics, Clarendon Laboratory, University of Oxford, Parks Road, Oxford OX1 3PU, United Kingdom

(Received 11 December 2009; revised manuscript received 25 November 2010; published 21 January 2011)

\begin{abstract}
Studies of materials under extreme conditions have relevance to a broad area of research, including planetary physics, fusion research, materials science, and structural biology with x-ray lasers. We study such extreme conditions and experimentally probe the interaction between ultrashort soft x-ray pulses and solid targets (metals and their deuterides) at the FLASH free-electron laser where power densities exceeding $10^{17} \mathrm{~W} / \mathrm{cm}^{2}$ were reached. Time-of-flight ion spectrometry and crater analysis were used to characterize the interaction. The results show the onset of saturation in the ablation process at power densities above $10^{16} \mathrm{~W} / \mathrm{cm}^{2}$. This effect can be linked to a transiently induced $\mathrm{x}$-ray transparency in the solid by the femtosecond $\mathrm{x}$-ray pulse at high power densities. The measured kinetic energies of protons and deuterons ejected from the surface reach several $\mathrm{keV}$ and concur with predictions from plasma-expansion models. Simulations of the interactions were performed with a nonlocal thermodynamic equilibrium code with radiation transfer. These calculations return critical depths similar to the observed crater depths and capture the transient surface transparency at higher power densities.
\end{abstract}

DOI: 10.1103/PhysRevE.83.016403

PACS number(s): 52.38.Kd, 52.38.Mf, 52.50.Jm

\section{INTRODUCTION}

Ultrashort and extremely intense photon pulses from x-ray free-electron lasers (FELs) have created a revolution in photon science. Breakthrough results have been reported in coherent diffractive imaging [1], femtosecond x-ray holography [2], cluster physics [3], and high-energy density science [4,5]. Parallel developments with table-top x-ray lasers demonstrate new capabilities at a significantly smaller laboratory scale [6-9].

The first years of research with short-wavelength FELs have demonstrated that at $\mathrm{x}$-ray frequencies, laser-matter interactions are significantly different from interactions at

\footnotetext{
${ }^{*}$ Present address: Department of Physics, Boston University, 590 Commonwealth Avenue, Boston, Massachusetts 02215, USA.

${ }^{\dagger}$ Present address: Center for Free-Electron Laser Science, DESY, Notkestraße 85, DE-22607 Hamburg, Germany.

${ }^{\ddagger}$ Present address: European XFEL GmbH, Albert-Einstein-Ring 19, DE-22761 Hamburg, Germany.

${ }^{\S}$ nicusor@xray.bmc.uu.se
}

optical frequencies [10-15]. Understanding and controlling parameters that govern extreme interactions between $\mathrm{x}$-ray radiation and materials are fundamentally important to many fields, including plasma acceleration and fusion research $[16,17]$ and the structure determination of single biomolecules [18], viruses, or cells [19].

An intense ultrashort $x$-ray pulse will destroy any sample in a process that spans a wide timescale. Direct photoionization is followed by cascade ionizations in 1-10 fs. Heating and thermalization of electrons follows, leading to electron-ion temperature equilibration in the sample within about $100 \mathrm{fs}$. The resulting plasma can reach very high temperatures, and in the present experiments $400 \mathrm{eV}$ (4.6 million $\mathrm{K})$ has been reached. The plasma expands over a couple of picoseconds and ends in the vaporization of micron-sized objects [2] or in the ablation of bulk solids (within a few nanoseconds) [4]. Resolidification of the surface layer in solids requires an additional $100 \mathrm{~ns}$ [20]. These processes are explored here in a combined experimental and theoretical study, using ultra-intense and ultrashort soft $\mathrm{x}$-ray pulses from the FLASH free-electron laser in Hamburg [21]. 


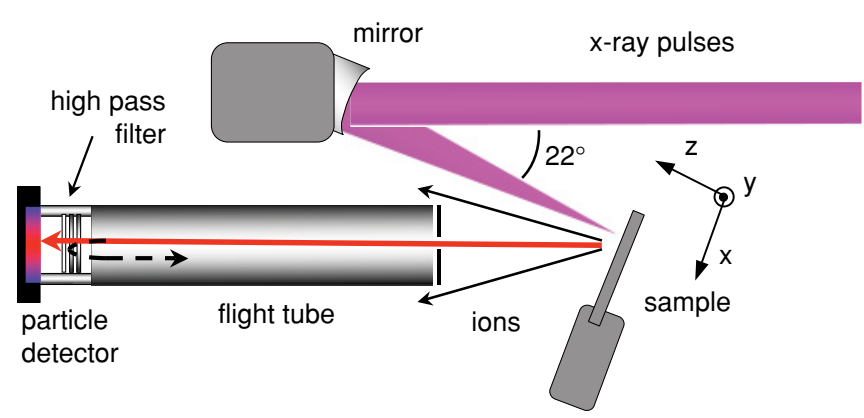

FIG. 1. (Color online) (a) Experimental setup. The x-ray FEL beam was focused by a mirror onto the sample at normal incidence. Each pulse ejected positive ions from the sample, and a fraction entered the flight tube of the ion spectrometer (horizontal thick solid red arrow). The high-pass filter selected high-energy ions, which hit the particle detector and generate flight-time spectra. Less energetic ions were deflected (dashed arrow).

\section{RESULTS}

Experimental measurements were performed at FLASH in April 2008 in single-pulse mode with a pulse length of about $15 \mathrm{fs}$ and at a wavelength of $13.5 \mathrm{~nm}$ (photon energy $\hbar \omega=92 \mathrm{eV}$ ). The average pulse energy was about $30 \mu \mathrm{J}$ with individual pulses varying between 7 and $70 \mu \mathrm{J}$. The energies of the individual pulses were measured using a gas monitor detector (GMD) [21]. The highly collimated beam was focused using a $\mathrm{Mo} / \mathrm{Si}$ multilayer-coated off-axis $\left(22^{\circ}\right)$ parabola with a focal length of $270 \mathrm{~mm}$ and an initial reflectivity of $67 \%$ (Fig. 1). The resulting submicron spot with a power density above $10^{17} \mathrm{~W} / \mathrm{cm}^{2}$ at best focus [5,22] produced well-defined craters in the solid samples [Figs. 2(a), 2(b), and 2(c)].

The samples were mounted with the focused FEL beam normal to the sample surface. The sample holder allowed movement of the sample in three orthogonal directions $(x, y$, and $z$ ), where $z$ denotes the axis along the focused FEL beam (Fig. 1). The power density on the sample was controlled by moving the sample through the focal plane of the mirror (along $z$ ) and by changing the energy of the individual pulse. At a selected average pulse energy, individual pulses have intrinsic variations due to the stochastic nature of the lasing process [21]. The power density for each individual exposure was given by the measured FEL pulse energy for that specific pulse, the calculated beam area at the sample surface, and the pulse duration.

Uncertainties in the measurements are due to errors in the GMD data (around 10\%) [23], to a decrease in the mirror reflectivity during this experiment (from 67\% to 50\%) [22], and to an uncertainty in the exact sample position $(z)$ relative to the focal plane. The uncertainty in the position affects calculated power densities by up to $30 \%$ when the sample was close to the best focus.

The profile of the focused beam was determined with attenuated pulses as described in Ref. [24]. When moving the sample out of focus, the profile of the beam [Fig. 2(a)] produced concentric imprints [Fig. 2(d)] originating from coherent scattering from a circular aperture upstream in the beam line. The simulated fringe pattern [Fig. 2(e)] shows a
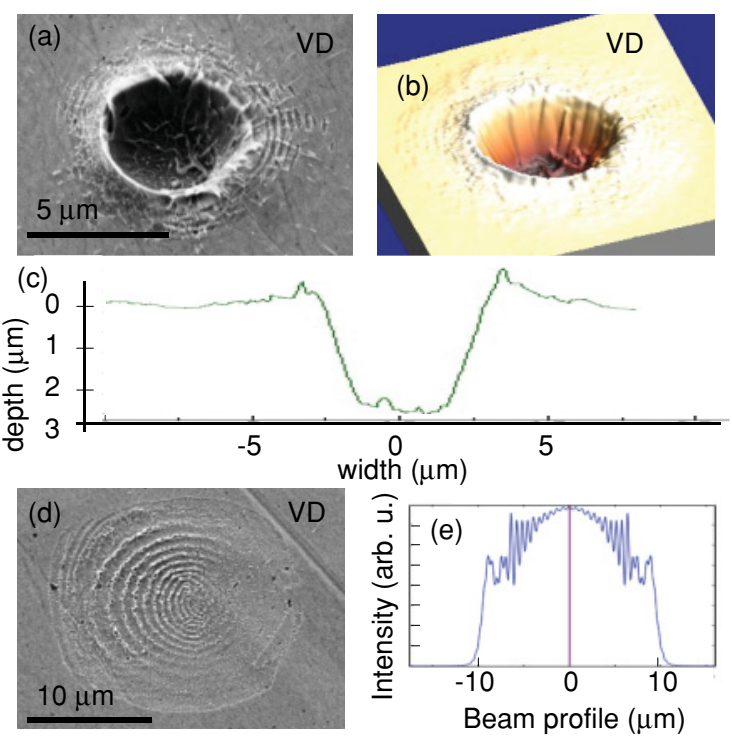

FIG. 2. (Color online) (a) Scanning electron microscopy image of a crater formed under in-focus conditions in deuterated vanadium (VD) by a single $13.5 \mathrm{~nm}$ FEL pulse at about $10^{17} \mathrm{~W} / \mathrm{cm}^{2}$. (b, c) Atomic force microscopy image and depth profile of the same crater. (d) Scanning electron microscopy image of a crater formed in VD under out-of-focus conditions (below $10^{16} \mathrm{~W} / \mathrm{cm}^{2}$ ). (e) Simulated out-of-focus beam profile with fringes caused by scattering off an off-center beam-line aperture.

striking similarity with the observed out-of-focus imprint on the sample [Fig. 2(d)].

The targets were bulk samples of niobium $(\mathrm{Nb})$ and vanadium (V). These metals are well-known hydrogen absorbers, and a number of samples were loaded with deuterium according to standard techniques [25]. The resulting deuterated niobium (NbD) and deuterated vanadium (VD) samples had deuterium/metal atomic ratios of $0.680 \pm 0.002$ and $0.564 \pm 0.002$, respectively, in the bulk. The V, VD, and NbD samples were $12 \times 12 \times 1 \mathrm{~mm}$ mechanically polished polycrystalline plates. The $\mathrm{Nb}$ sample was a $10 \times 10 \times 3 \mathrm{~mm}$ electropolished single crystal. The position of best focus was found for all samples except V. Accordingly, power densities above $10^{17} \mathrm{~W} / \mathrm{cm}^{2}$ were reached for all samples except $\mathrm{V}$. At the time of the FLASH measurements the deuterium was depleted from the sample surface. All samples had impurities in the surface layer, notably hydrogen, a fact routinely exploited in laser-driven proton-acceleration experiments [26]. This provides an opportunity to separate surface and bulk effects in the processes of crater formation and acceleration of light ions (protons $\mathrm{H}^{+}$and deuterons $\mathrm{D}^{+}$).

Ion time-of-flight (TOF) spectra were recorded by a TOF instrument [27] mounted on axis with the FEL beam with a micro channel plate (MCP) detector located about $600 \mathrm{~mm}$ from the interaction region (Fig. 1). The detector assembly was equipped with a high-pass (HP) filter in the form of metal grids in front of the MCP detector (Fig. 1). When a high positive voltage was applied to these grids, positive ions with low energy-to-charge ratios were repelled and did not reach the MCP. No other electric or magnetic fields were used to influence the flight paths of the ions, and thus 


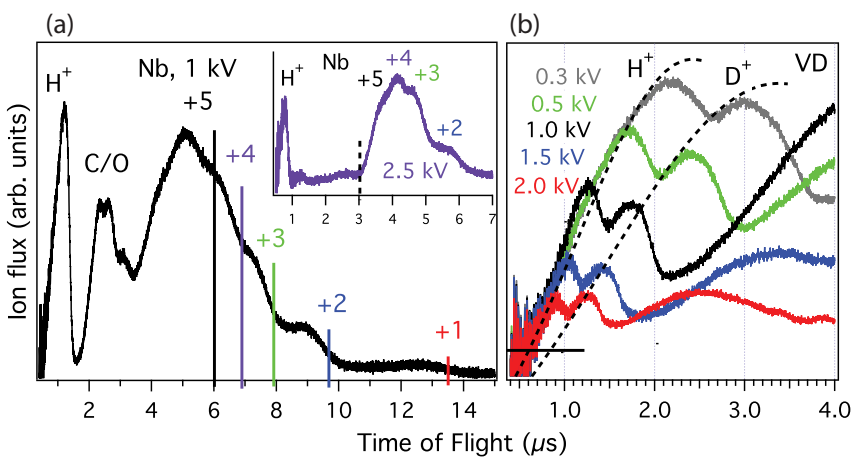

FIG. 3. (Color online) Averaged ion time-of-flight (TOF) spectra. (a) Entire TOF spectrum obtained from niobium $(\mathrm{Nb})$ with a high-pass (HP) filter setting of $1 \mathrm{kV}$ (inset shows result for HP filter at $2.5 \mathrm{kV}$ ). (b) Ion TOF spectra of the light ions $\left(\mathrm{H}^{+}\right.$and $\left.\mathrm{D}^{+}\right)$ obtained from deuterated vanadium (VD) with different settings on the HP filter $(0.3$ to $2.0 \mathrm{kV}$, shown from top down with solid lines). Dotted lines follow the envelope for highest energies; the solid horizontal line shows a $95 \%$ confidence level for signal above background.

the detector records only the ions ejected into the narrow solid angle covered by the MCP, and the observed ion energies represent directly the ion acceleration processes in the interaction region. Due to the shot-to-shot fluctuation in pulse intensity, the spectra were averaged over at least 30 shots for each experimental setting. All assignments of features in TOF spectra are supported by simulations of ion flights performed with SIMION [28] for the specific experimental geometry.

The measured ion TOF spectra from $\mathrm{Nb}$ and VD display a number of general features (Fig. 3). Metal ions with charge states up to +5 are observed with flight times longer than $3 \mu$ s. Multiply-charged ions with keV energies have previously been observed from exposure of bulk semiconductors to extreme ultraviolet pulses [29]. At flight times between 0.5 and $4 \mu \mathrm{s}$ we observe hydrogen, oxygen, and carbon ions present in surface contaminations. This surface acceleration is similar to the proton acceleration from thin metal films using visible lasers [26]. In this flight-time region we could also observe $\mathrm{D}^{+}$when deuterated samples were exposed [Fig. 3(b)].

Under the influence of selected HP filter settings, and in comparison with detailed flight-time simulations, element and charge-state specific assignments can be done, and maximum observed ion energies can be determined. For a HP filter setting of $1 \mathrm{kV}$, ions with kinetic energies less than $q \times 1 \mathrm{keV}$ (where $q$ is the charge state of the ion) were deflected by the filter, resulting in sharp cutoffs suppressing the long flight-time (low-energy) part of the ion populations. In the flight-time spectrum obtained from $\mathrm{Nb}$, these cutoffs are seen between 6 and $14 \mu \mathrm{s}$ for $\mathrm{Nb}$ ions with charge states +5 to +1 [vertical lines in Fig. 3(a)]. We observe that the different charge state populations have different high-energy profiles with highly charged metal ions more prominently present at short flight times [Fig. 3(a) and inset]. This shows that ion acceleration is affected by charge separation. Increasing the grid voltage to $2.5 \mathrm{kV}$ shifted the cutoffs to shorter flight times, as expected [Fig. 3(a) inset]. Further, the peaks associated with $\mathrm{C}$ and $\mathrm{O}$ ions are suppressed, showing that no significant fraction of these ions obtained energies in excess of $2.5 \mathrm{keV}$. With the suppression of the $\mathrm{C}$ and $\mathrm{O}$ peaks the high-energy end of the spectrum of the $\mathrm{Nb}$ ions becomes detectable at a flight time of $3 \mu$ s [dashed vertical line in Fig. 3(a) inset], corresponding to a kinetic energy of about $20 \mathrm{keV}$.

Deuterons were detected together with protons only when deuterated samples were moved during exposure in the $x$ direction in such a way that each pulse hit partly in the crater formed by the previous pulse (with a repetition rate of $5 \mathrm{~Hz}$ ). No, or marginal, deuterium signals were recorded when samples were moved in $-x, y$, or $-y$ directions. When the samples were moved in the $x$ direction, part of the exposed crater surface was normal to the MCP detector [Figs. 1(a) and 3(b)]. Assuming that the majority of the ions are ejected normal to the surface, this explains the selective observation of deuterons. Furthermore, when a pulse hits partly in the crater formed $200 \mathrm{~ms}$ before, deuterium is present in the freshly ablated crater surface and available for surface acceleration from the following FEL pulse. This shows that acceleration of light ions to high energies is indeed a surface effect. The observation of protons was not affected by this anisotropy. We attribute this to the fact that protons were accelerated from the flat surface surrounding the craters. These areas have the same angle with respect to the location of the ion detector regardless of the direction of the sample movement ( $22^{\circ}$ off-normal).

When $\mathrm{H}^{+}$and $\mathrm{D}^{+}$were simultaneously observed they had similar high-energy distributions [Fig. 3(b)] with maximum detectable energies of about $5 \mathrm{keV}$ (Fig. 4), corresponding to flight times of about 0.6 and $0.85 \mu$ s for $\mathrm{H}^{+}$and $\mathrm{D}^{+}$, respectively. The present results indicate that directional proton/deutreron acceleration can be achieved by optimizing the experimental geometry using targets such as thin films [26] or microstructures [30].

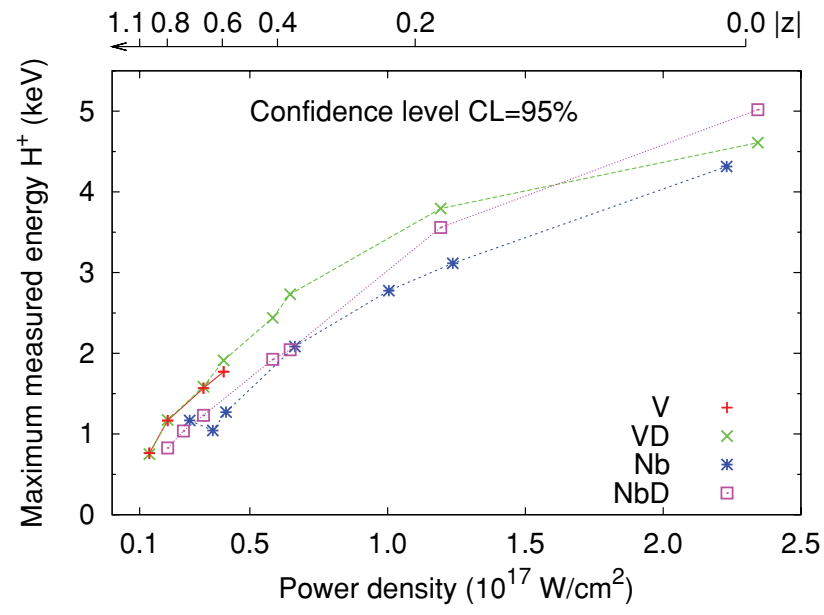

FIG. 4. (Color online) The highest observed proton energies as a function of power density for various solids. Measurements were performed at an average pulse intensity of $30 \mu \mathrm{J}$ at $13.5 \mathrm{~nm}$ wavelength. The change in power density was obtained from a variable focus, by moving the sample along the $z$ direction [Fig. 1(a); shown on the top horizontal axis, in mm relative to best focus]. The confidence level for detection of signal above the background is at $95 \%$. 


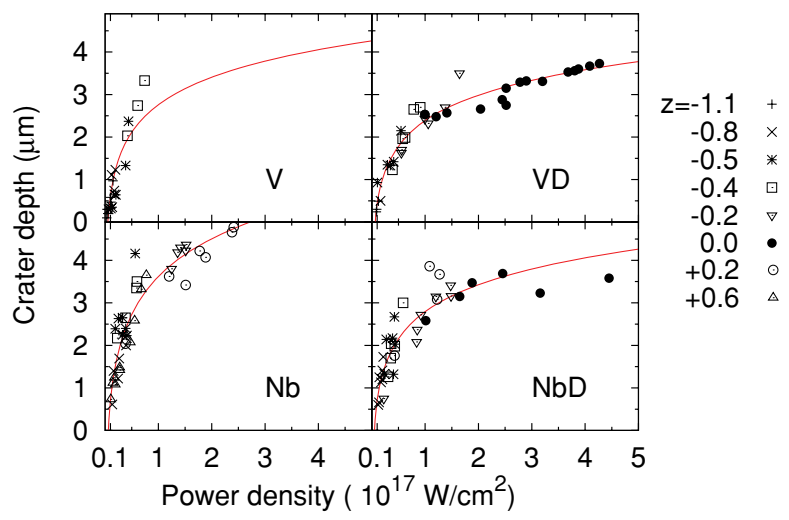

FIG. 5. (Color online) Crater depth as a function of power density of the incident $\mathrm{x}$-ray pulse measured using atomic force microscopy. Different power densities in single shots are due to both FEL pulse energy fluctuations and moving the sample through the focus ( $z$ shows position of the sample in $\mathrm{mm}$ relative to best focus). The solid red line shows a fit of the function $\ln I^{n}$, with $n=0.9$ for polycrystalline samples $(\mathrm{V}, \mathrm{VD}, \mathrm{NbD})$ and $n=1.3$ for single-crystal $\mathrm{Nb}$.

As the power density increased above $10^{16} \mathrm{~W} / \mathrm{cm}^{2}$ we observed a saturation in the highest detectable proton energies (Fig. 4). This saturation becomes particularly clear above $10^{17} \mathrm{~W} / \mathrm{cm}^{2}$ in the samples where these power densities is reached. We show later (Fig. 8 and discussion) that saturation can be described in terms of rapid inner-shell processes resulting in a reduction in the surface absorption and hence a less efficient proton acceleration. For $92 \mathrm{eV}$ photons vanadium has 5 times higher cold absorption than niobium (corresponding attenuation lengths in crystals are about $40 \mathrm{~nm}$ and $200 \mathrm{~nm}$, respectively) [31]. This is reflected in our data since, at low power densities, the protons accelerated from the $\mathrm{V}$ and VD surfaces were observed to have higher energies than those accelerated from $\mathrm{Nb}$ and $\mathrm{NbD}$. Increasing the power density, the protons acceleration from VD saturated more rapidly than from $\mathrm{Nb}$ and $\mathrm{NbD}$.

Following the exposure, individual craters formed by the laser pulses were characterized by scanning electron microscopy (SEM) [Figs. 2(a) and 2(d)] and tapping mode atomic force microscopy (AFM) [Figs. 2(b) and 2(c)]. Craters formed under in-focus conditions were generally deep and narrow with rim to rim diameters down to about $5 \mu \mathrm{m}$ and depths commonly exceeding $3 \mu \mathrm{m}$ with a largest observed crater depth of $4.8 \mu \mathrm{m}$. An increase in power density causes a gradual increase of the observed crated depth in all samples (Fig. 5). Changes in the observed crater depth are similar for changes in power density caused either by different pulse energies or by moving the sample with respect to the focus. Saturation in the measured crater depth is observed from $10^{16} \mathrm{~W} / \mathrm{cm}^{2}$ onward and becomes significant with power densities exceeding $10^{17} \mathrm{~W} / \mathrm{cm}^{2}$ in the samples where such conditions are reached.

\section{DISCUSSION}

Theoretical modeling of the laser-matter interaction and plasma dynamics is employed for comparison with the observed crater depths and ion energies. To calculate the rapid change in ion and electron temperatures due to the ionization induced by the intense pulse, we use a previously developed approach [19,32]. We use a multidimensional nonlocal thermodynamic equilibrium (non-LTE) radiation transfer code CRETIN [33], based on a plasma formulation originally developed for low-density hot matter [34,35]. CRETIN can be used to calculate level populations and transition rates and provides absorption, heating rates, and conduction coefficients for each time step of the simulation. For this study we use screened hydrogenic atomic models, similar to those described by More [36]. The calculated cold absorption spectra of $\mathrm{V}$ and $\mathrm{Nb}$ have been scaled to fit the Henke absorption coefficients [31] at $13.5 \mathrm{~nm}$. Heating is modeled in one dimension only, considering the laser spot size to be larger than the energy penetration depth in the sample, an approximation that may eventually break down when tighter focus is achieved. The depth over which the interaction is modeled is divided into 250 zones (4-40 nm thick) that exchange energy through radiation transport and electron thermal conduction. The time evolution is done in steps of $0.01 \mathrm{fs}$, and the total simulation length is $1 \mathrm{ps}$. The temporal profile of the pulse is assumed to be Gaussian with 15 fs FWHM. Earlier studies show that the pulse time structure has a minor impact on the ionization rates in the sample [32].

Nonthermal melting and ablation occur on timescales faster than conventional melting, an idea supported by earlier damage studies done at FLASH [4]. We define the critical charge density as the point where the density of free carriers $(N)$ is high enough to destabilize the lattice. This happens at $N \approx 10^{22} \mathrm{~cm}^{-3}$, and considering the atomic ratios and crystal densities this corresponds to an average ionization state per atom of $\bar{z}=0.14$ for $\mathrm{V}, \bar{z}=0.16$ for $\mathrm{VD}, \bar{z}=0.16$ for $\mathrm{Nb}$, and $\bar{z}=0.20$ for NbD. To check the impact of the initial heating on the damage process we simulate the time evolution of the critical charge density (Fig. 6) and compare it to the observed crater depth (Fig. 5). After 1 ps the simulated critical depth approaches the measured crater depths for power densities around $10^{16} \mathrm{~W} / \mathrm{cm}^{2}$ (Fig. 5). At the same time this charge density underestimates the crater depth between $10^{16}$ and $10^{17} \mathrm{~W} / \mathrm{cm}^{2}$ and overestimates it above $10^{17} \mathrm{~W} / \mathrm{cm}^{2}$.

We have looked at the initial heating process, which develops faster than plasma expansion [2] when matter transport during hydrodynamic expansion becomes significant. CRETIN also models the hydrodynamics using the equation of states from the atomic kinetics. At $10^{17} \mathrm{~W} / \mathrm{cm}^{2}$, we find that after $1 \mathrm{ps}$ the expansion of the zones at the surface leads to about $10 \%$ decrease in both the electron temperature $T_{e}$ and the average ionization in the surface zones. At the same time in the bulk, compression of zones due to high pressure leads to an increase of up to $20 \%$ in $T_{e}$ and $\bar{z}$. However, this shockwave takes longer than $1 \mathrm{ps}$ to propagate to $\mu \mathrm{m}$ depth, where it would be relevant to the ablation depth at the power densities studied here. We propose that hydrodynamics expansion on timescales longer than 1 ps contributes to crater formation following the initial heating process and is part of the reason why the critical charge density underestimates the crater depth at $1 \mathrm{ps}$ for power densities between $10^{16}$ and $10^{17} \mathrm{~W} / \mathrm{cm}^{2}$.

The heating of the sample is influenced by radiation transport and thermal electron conductivity. The dominant 
mechanism for the initial heating is radiation transport through the sample of both the initial laser pulse and the reemitted radiation. At the same time, electron thermal conduction will lead to a fast equilibration of the electron temperature between the different regions as well as a rapid cooling of the exposed surface. Any change in the opacity of the material during and after the pulse will affect radiation transport. In the case of high-intensity pulses, reduction of the opacity at the surface causes a more efficient transfer of energy into the depth of the sample. From the observed saturation for the proton acceleration (Fig. 4) we have experimental evidence of a surface transparency, which becomes increasingly significant for power densities above $10^{17} \mathrm{~W} / \mathrm{cm}^{2}$. This onset of surface transparency is likely linked to the fast increase in distance from the sample surface for the critical charge density (ablation depth) as the power density approaches $10^{17} \mathrm{~W} / \mathrm{cm}^{2}$ (Fig. 6). However, this increase does not have full effect in the crater formation since as the craters get deeper the deposited energy is diluted into a larger volume and more radiation is lost through the sides, resulting in an observed saturation in the crater depth for power densities above $10^{17} \mathrm{~W} / \mathrm{cm}^{2}$ (Fig. 5).

An alternative to the criterion of critical depth based on free carriers is to use the experimental measurements to determine the critical temperature for the equation of state in a two-phase vaporization model [23]. In this approach the measured crater depths correspond to simulated electron temperatures of about $0.27-0.35 \mathrm{eV}$ for $\mathrm{V}$ and $0.29-0.36 \mathrm{eV}$ for $\mathrm{VD}$, after the $\mathrm{x}$-ray pulse ends. For $\mathrm{Nb}$ and $\mathrm{NbD}$ samples the critical temperature is higher, reaching $0.30-0.65 \mathrm{eV}$ for $\mathrm{Nb}$ and $0.45-0.70 \mathrm{eV}$ for $\mathrm{NbD}$. These temperatures were obtained from the interval of power densities shown in Fig. 5 and are consistent with the range of critical temperatures in solids, obtained at lower power densities [23].

Our simulations follow the thermalization process in the dense hot plasma created by the exposure [Fig. 7(a)]. The equilibration timescale of the electron and ion temperature

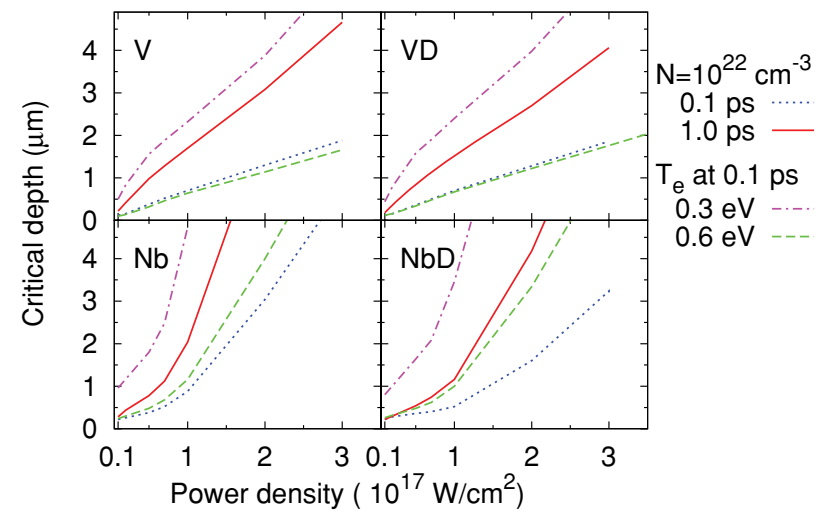

FIG. 6. (Color online) Simulated critical depth as a function of power density of the incident x-ray pulse for different materials. Simulations are performed with CRETIN and show the sample depth where electron density reaches the critical value $N \approx 10^{22} \mathrm{~cm}^{-3}$, presented with dotted blue lines after $0.1 \mathrm{ps}$ and solid red lines after $1 \mathrm{ps}$. The depths where the electron temperature reaches $T_{e}=0.3 \mathrm{eV}$ (dash-dotted purple lines) and $T_{e}=0.6 \mathrm{eV}$ (dashed green lines) after $0.1 \mathrm{ps}$ are also shown. The x-ray pulse is $15 \mathrm{fs}$ FWHM and $13.5 \mathrm{~nm}$ wavelength. (a)

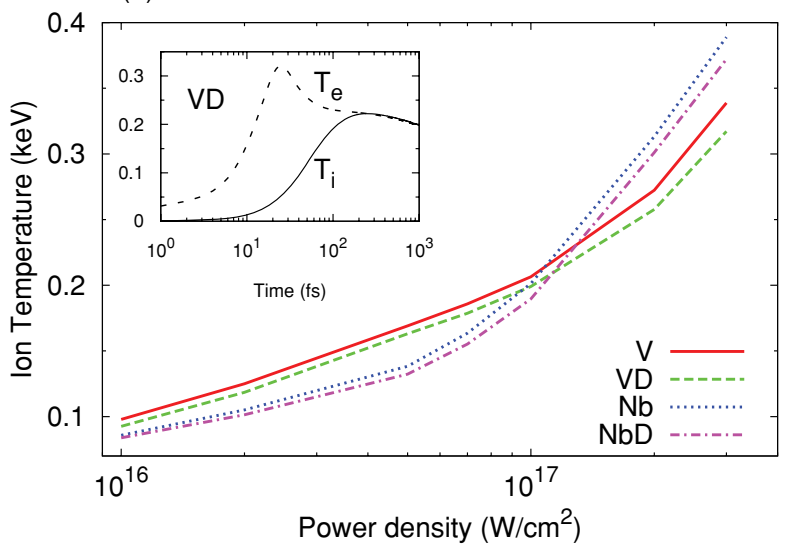

(b)

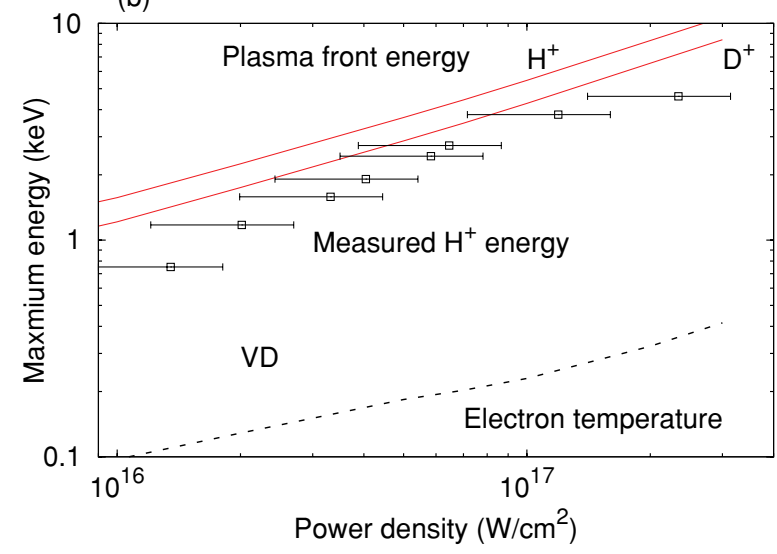

FIG. 7. (Color online) (a) Simulated ion temperature at the plasma surface $1 \mathrm{ps}$ after the pulse as a function of power density. The inset shows the equilibration of the electron and ion temperatures in VD following illumination with a $15 \mathrm{fs}$ soft x-ray pulse of $13.5 \mathrm{~nm}$ and a power density of $10^{17} \mathrm{~W} / \mathrm{cm}^{2}$. (b) The highest measured proton energies in VD (boxes, confidence level 95\%) compared with the simulated electron temperature at the surface (dashed line) and the calculated energy $E_{\max }$ (Eq. (1)) of plasma front expansion for light ions (red solid lines).

is about 100 fs [Fig. 7(a) inset], and the plasma reaches temperatures between 100 and $400 \mathrm{eV}$ at power densities above $10^{16} \mathrm{~W} / \mathrm{cm}^{2}$. The acceleration of ions in the plasma front can be modeled using a self-similar isothermal fluid model for plasma expansion into vacuum [37]. This models charge separation and has been shown to give a very good description of the laser-driven acceleration of protons [26] over a wide range of laser parameters in the optical regime when the acceleration time $t_{\mathrm{acc}}=1.3 \times \mathrm{FWHM}$ of the laser pulse. The maximum energy gained by the accelerated ions $\left(E_{\max }\right)$ depends on the charge number $Z$, plasma frequency $\omega_{\mathrm{pi}}$, electron temperature $T_{e}(\mathrm{eV})$, and $t_{\mathrm{acc}}$, as

$$
E_{\max }=2 Z T_{e}\left[\ln \left(\frac{\omega_{\mathrm{pi}} t_{\mathrm{acc}}}{2 \sqrt{2}}\right)-1\right]^{2}
$$

In the range of power densities studied here, $1 \times$ $10^{16}-2 \times 10^{17} \mathrm{~W} / \mathrm{cm}^{2}$, the maximum detected energy of 
(a)

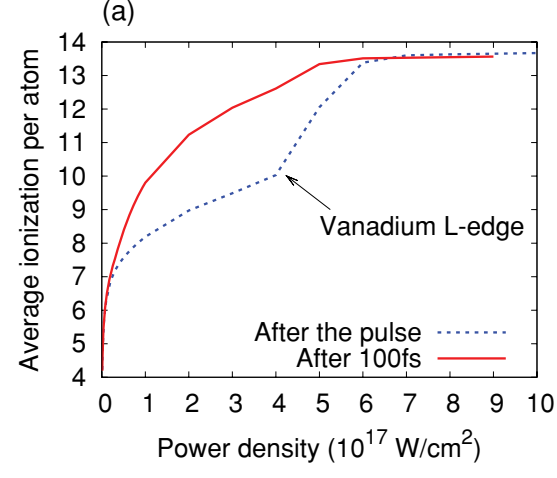

(b)

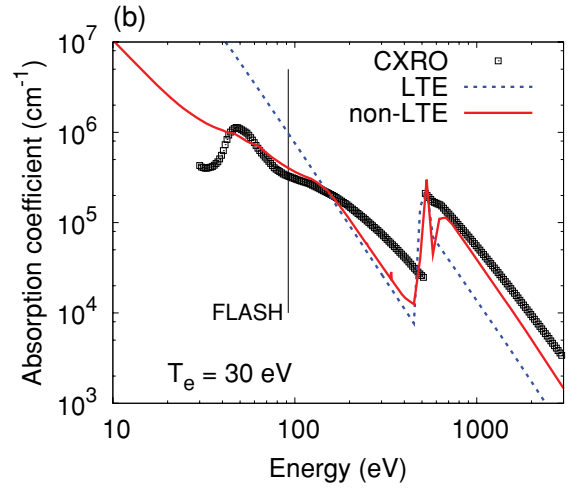

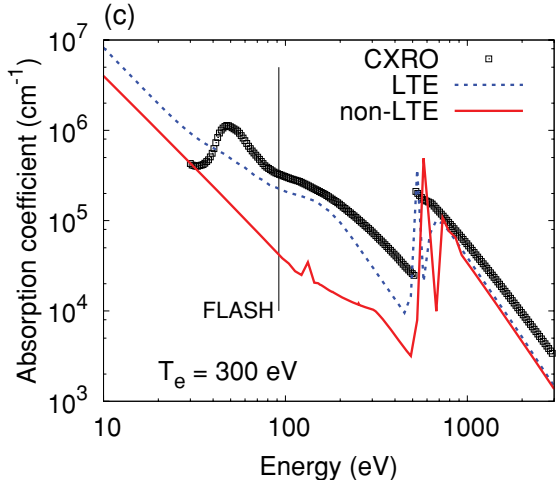

FIG. 8. (Color online) (a) Simulated average ionization per atom at the VD surface as a function of power density. At power densities above $4 \times 10^{17} \mathrm{~W} / \mathrm{cm}^{2}$, the electron temperature reaches the $500 \mathrm{eV}$ level of the L edge in vanadium. (b, c) Absorption coefficient as a function of photon energy, for the case of cold material (data from The Center for X-ray Optics, CXRO) [31], local thermodynamic equilibrium (LTE), and nonlocal thermodynamic equilibrium (non-LTE), for two different electron temperatures. At high electron temperatures, the non-LTE model leads to a substantial drop in absorption. Electron temperatures of $300 \mathrm{eV}$ are obtained in the sample during the FEL pulse, and a drop in absorption will lead to transparency of the hotter zones of the material and to deeper propagation of radiation into the bulk.

the accelerated protons and deuterons reaches values between 1 and $5 \mathrm{keV}$ [Fig. 7(b)], approaching the simulated plasma front energy $E_{\max }$ (Eq. (1)) around $10^{17} \mathrm{~W} / \mathrm{cm}^{2}$. Furthermore, the highly charged $\mathrm{Nb}$ and $\mathrm{V}$ ions $(5+)$ are expected to have energies from 4 to $40 \mathrm{keV}$ according to the plasma front expansion predictions, in line with our maximum observed energies of $20 \mathrm{keV}$.

Our analyses of the highest observed proton energies (Fig. 4) and crater depth (Fig. 5) show saturation effects. CRETIN provides an ideal tool for investigating the dynamics of saturation and transparency at the surface of solids. Fig. 8(a) shows the saturation of the ionization level at the surface of the plasma as a function of the power density. A similar effect can also be seen in the electron temperature, providing the mechanism for the observed saturation of the plasma expansion and the energy of the expanding plasma front (Fig. 4). Figure 8(a) features the ionization L edge of vanadium at $500 \mathrm{eV}$, which is reached under conditions when the electron temperature is high enough for collisional ionizations to be significant. Figures 8(b) and 8(c) show how transparency arises at the surface of the sample, as the electron temperature increases to $300 \mathrm{eV}$, a temperature reached during the pulse duration [Fig. 7(a) inset]. The driving mechanism is the non-LTE dynamics. This is strongly correlated with the plasma temperature, which is controlled by the power density of the incident laser [Fig. 7(a)].

Previous studies in the soft x-ray regime [32] have shown that LTE dynamics calculations overestimate the average ionization, leading to enhanced inverse bremsstrahlung absorption and higher opacity at the surface. In the present study we find that non-LTE dynamics is necessary to model the observed transparency induced surface saturation and gives a better description of the ionization. Figures 8(b) and 8(c) show the absorption spectra for the two models, where the non-LTE approach gives a considerably lower opacity for both a warm plasma (30 eV electron temperature) and hot plasma (300 eV electron temperature). During a high-power-density FLASH pulse where a high electron temperature is achieved [e.g., Fig. 7(a) inset], the surface gets more transparent to radiation and the incident laser pulse, and reemitted radiation is transported into the bulk.

\section{CONCLUSION}

Focused to a micrometer spot, individual femtosecond pulses from the FLASH free-electron laser exposed the metal samples to power densities above $10^{17} \mathrm{~W} / \mathrm{cm}^{2}$, producing a hot dense plasma with temperatures reaching $400 \mathrm{eV}$ (4.6 million $\mathrm{K}$ ). Under these extreme conditions the initial heating induced ablation several $\mu \mathrm{m}$ into the sample. Subsequently, a plasma expansion into vacuum with charge separation ejected ions with kinetic energies reaching $5 \mathrm{keV}$ for protons and deuterons and $20 \mathrm{keV}$ for multiply-ionized metal ions. With increasing power densities we observe saturation effects (Figs. 4 and 5) related to a transient surface transparency that develops in the sample during the pulse. Transparency leads to a dilution of the deposited energy into a larger volume, resulting in a less efficient ion acceleration mechanism from the sample surface and saturation in the observed crater depths.

\section{ACKNOWLEDGMENTS}

The authors give special thanks to Howard A. Scott for valuable input regarding CRETIN. This work was supported by the following agencies: the Swedish Research Council through a Centre of Excellence Award to JH, the Virtual Institute Program of the Helmoltz Association (VH-VI-302), the DFG Cluster of Excellence at the Munich Centre for Advanced Photonics, the Portuguese Science and Technology Foundation, the Czech Ministry of Education (LC510, LC528, ME10046, and LA08024) and Academy of Sciences (AV0Z10100523, IAAX00100903, and KAN300100702), and the MSHE of Poland, SPB No. DESY/68/2007. Computations were performed on UPPMAX under project p2009018. SEM measurements were performed at the Microscopy and Microanalysis Group, Chalmers University of Technology. Access to FLASH was supported by the European Union under contract RII3-CT-2004-506008 (IA-SFS). 
[1] H. N. Chapman et al., Nature Phys. 2, 839 (2006).

[2] H. N. Chapman et al., Nature (London) 448, 676 (2007).

[3] C. Bostedt et al., Phys. Rev. Lett. 100, 133401 (2008).

[4] J. Chalupsky et al., Opt. Express 17, 208 (2009).

[5] B. Nagler et al., Nature Phys. 5, 693 (2009).

[6] P. Zeitoun et al., Nature (London) 431, 426 (2004).

[7] Y. Wang, E. Granados, F. Pedaci, D. Alessi, B. Luther, M. Berrill, and J. J. Rocca, Nat. Phot. 2, 94 (2008).

[8] A. Ravasio et al., Phys. Rev. Lett. 103, 028104 (2009).

[9] M. Fuchs et al., Nature Phys. 5, 826 (2009).

[10] A. A. Sorokin, S. V. Bobashev, T. Feigl, K. Tiedtke, H. Wabnitz, and M. Richter, Phys. Rev. Lett. 99, 213002 (2007).

[11] H. Wabnitz et al., Nature (London) 420, 482 (2002).

[12] S. P. Hau-Riege, R. A. London, H. N. Chapman, and M. Bergh, Phys. Rev. E 76, 046403 (2007).

[13] S. Vinko et al., Phys. Rev. Lett. 104, 225001 (2010).

[14] J. P. Cryan et al., Phys. Rev. Lett. 105, 083004 (2010).

[15] L. Young et al., Nature (London) 466, 56 (2010).

[16] T. Ditmire, J. Zweiback, V. P. Yanovsky, T. E. Cowan, G. Hays, and K. B. Wharton, Nature (London) 398, 489 (1999).

[17] M. Roth et al., Phys. Rev. Lett. 86, 436 (2001).

[18] R. Neutze, R. Wouts, D. van der Spoel, E. Weckert, and J. Hajdu, Nature (London) 406, 752 (2000).

[19] M. Bergh, G. Huldt, N. Timneanu, F.R.N.C. Maia, and J. Hajdu, Q. Rev. Biophys. 41, 181 (2008).

[20] M. Harbst, T.N. Hansen, C. Caleman, W.K. Fullagar, P. Jönsson, P. Sondhauss, O. Synnergren, and J. Larsson, Appl. Phys. A 81, 893 (2005).
[21] K. Tiedtke et al., New J. Phys. 11, 023029 (2009).

[22] A. J. Nelson et al., Opt. Express 17, 18271 (2009).

[23] S. P. Hau-Riege et al., Appl. Phys. Lett. 90, 173128 (2007).

[24] J. Chalupsky et al., Opt. Express 15, 6036 (2007).

[25] G. K. Palsson, A. R. Rennie, and B. Hjorvarsson, Phys. Rev. B 78, 104118 (2008).

[26] J. Fuchs et al., Nature Phys. 2, 48 (2006).

[27] R. Sobierajski et al., Rev. Sci. Instrum. 76, 013909 (2005).

[28] D. Dahl, Int. J. Mass Spectrom. 200, 3 (2000).

[29] J. Krzywinski et al., J. Appl. Phys. 101, 043107 (2007).

[30] H. Schwoerer, S. Pfotenhauer, O. Jäckel, K.-U. Amthor, B. Liesfeld, W. Ziegler, R. Sauerbrey, K. W. D. Ledingham, and T. Esirkepov, Nature (London) 439, 445 (2006).

[31] B. Henke, E. Gullikson, and J. Davis, Atom. Nucl. Data Tabl. 54, 181 (1993).

[32] M. Bergh, N. Tîmneanu, S. P. Hau-Riege, and H. A. Scott, Phys. Rev. E 77, 026404 (2008).

[33] H. Scott, J. Quant. Spectrosc. Radiat. Transfer 71, 689 (2001).

[34] M. Fajardo, P. Zeitoun, and J. Gauthier, Eur. J. Phys. D 29, 69 (2004).

[35] K. Eidmann, J. Meyer-ter-Vehn, T. Schlegel, and S. Huller, Phys. Rev. E 62, 1202 (2000).

[36] R. More, J. Quant. Spectrosc. Radiat. Transfer 27, 345 (1982).

[37] P. Mora, Phys. Rev. Lett. 90, 185002 (2003). 